\title{
Technetium-99m-Labeled Autologous Serum Albumin: A Personal-Exclusive Source of Serum Component
}

\author{
Yuh-Feng Wang, ${ }^{1,2}$ Yi-Chun Chen, ${ }^{1,3}$ Dian-Kun Li, ${ }^{1,4}$ and Mei-Hua Chuang ${ }^{5}$ \\ ${ }^{1}$ College of Medicine, Tzu Chi University, Hualien 97004, Taiwan \\ ${ }^{2}$ Department of Nuclear Medicine, Buddhist Dalin Tzu Chi General Hospital, Chiayi 62247, Taiwan \\ ${ }^{3}$ Division of Nephrology, Department of Internal Medicine, Buddhist Dalin Tzu Chi General Hospital, Chiayi 62247, Taiwan \\ ${ }^{4}$ Department of Hematology and Oncology, Buddhist Dalin Tzu Chi General Hospital, Chiayi 62247, Taiwan \\ ${ }^{5}$ Department of Pharmacy, Buddhist Dalin Tzu Chi General Hospital, Chiayi 62247, Taiwan
}

Correspondence should be addressed to Yuh-Feng Wang, yuhfeng@gmail.com

Received 31 December 2010; Accepted 3 March 2011

Academic Editor: Lie-Hang Shen

Copyright (c) 2011 Yuh-Feng Wang et al. This is an open access article distributed under the Creative Commons Attribution License, which permits unrestricted use, distribution, and reproduction in any medium, provided the original work is properly cited.

\begin{abstract}
Technetium-99m human serum albumin $\left({ }^{99} \mathrm{~m} \mathrm{Tc}-\mathrm{HSA}\right)$ is an important radiopharmaceutical required in nuclear medicine studies. However, the risk of transfusion-transmitted infection remains a major safety concern. Autopreparation of serum component acquired from patient provides a "personal-exclusive" source for radiolabeling. This paper is to evaluate the practicality of onsite elusion and subsequent radiolabeling efficacy for serum albumin. Results showed that the autologous elute contained more albumin fraction than serum without extraction procedure. Good radiochemical purity and stability were demonstrated after radiolabeling. Biodistribution study showed that labeled albumin accumulated immediately in the lung, liver, and kidney. It was cleared steadily and excreted in the urine. The biologic half-life was defined, and all samples passed the pyrogenicity and sterility tests. In conclusion, autoalbumin could be extracted and radiolabeled properly in a nuclear medicine setting. Moreover, the risk of transfusion-transmitted infection associated with nonautologous, multisource ${ }^{99 \mathrm{~m}} \mathrm{Tc}-\mathrm{HSA}$ agents can be reduced.
\end{abstract}

\section{Introduction}

Technetium-99m-labeled human serum albumin $\left({ }^{99 \mathrm{~m}} \mathrm{Tc}-\right.$ HSA) is an important radiopharmaceutical commonly used in nuclear medical services. It is routinely used in lymphoscintigraphic studies, sentinel lymph node mapping, blood pool imaging, and protein-losing gastroenteropathy [1-7]. However, preparing and maintaining stocks of ${ }^{99 \mathrm{~m}} \mathrm{Tc}-\mathrm{HSA}$ remain a challenge due to a shortage in the supply of commercially available kits and a lack of awareness regarding unknown transfusion-induced infectious diseases.

HSA is the major protein component of human plasma and is crucial for maintaining plasma volume and the osmotic pressure of circulating blood [8]. Plasma-derived HSA (pHSA) is used clinically to correct circulating plasma volume and improve colloid osmotic pressure. Nevertheless, the pHSA supply is limited as it is manufactured from a fractionation of pooled, donated human plasma. This nonautologous, multisource approach introduces significant risks of allergic reactions and contamination by bloodderived pathogens, such as human immunodeficiency virus (HIV), viral hepatitis, and Creutzfeldt-Jakob disease prions $[9,10]$. Since ${ }^{99 \mathrm{~m}} \mathrm{Tc}-\mathrm{HSA}$ is widely applied to nuclear medicine techniques, constant and stable sources of albumin are necessary to sustain these diagnostic procedures.

Serum products created from a single-source or limited domestic supplies are safer than those obtained from pooled commercial sources, according to the World Health Organization (WHO) [11-13]. In our previous study, a protocol was formulated using a domestic serum product to prepare ${ }^{99 \mathrm{~m}} \mathrm{Tc}-\mathrm{HSA}$ on site in a nuclear medicine laboratory [14]. This preparation proved safer than the commercially supplied pooled serum products, biologically stable, and clinically effective.

However, the risks of introducing an unknown infectious agent could not be completely eliminated. Therefore, 
a need for a protocol to create a single-source autologous serum albumin (autoalbumin) still exists. Autologous blood donation is the safest means by which clinical transfusion products can be prepared for an individual $[15,16]$. In this article, we describe the development of a ${ }^{99 \mathrm{~m}} \mathrm{Tc}-\mathrm{HSA}$ preparation technique using autologous serum elution, the ${ }^{99 \mathrm{~m}} \mathrm{Tc}-$ autoalbumin. In addition to labeling efficiency and stability studies, we performed in vivo biodistribution and biological half-life studies and tested sterility and pyrogenicity.

\section{Materials and Methods}

2.1. Extraction of Albumin from Serum. Fresh serum was collected following centrifugation (3,500 rpm for 5 minutes) of single-source blood samples from healthy volunteers. Affinity chromatographic columns, which is cross-linked agarose gel with covalently coupled Cibacron Blue [17] (Aurum Affi-Gel Blue column; Bio-Rad catalog no. 7326708 , CA, USA), were prepared by washing with low-salt $20 \mathrm{mM}$ Tris buffer solution ( $\mathrm{pH} \mathrm{8.3)}$ and desiccated in a microcentrifuge (7,000 rpm for 5 seconds). Serum samples were prepared by dilution with Tris buffer solution at $1: 3$. Serum sample $(800 \mu \mathrm{L})$ was then added to the column, and the column was gently vortexed for 5 to 10 minutes. The column was allowed to drain and washed twice with Tris buffer solution. All flow-through was discarded, and the serum protein remained bound to the column's resin beads. Two elution buffer solutions were used to remove the protein fraction from the bead.

Preparation 1 (Pre-1). Laemmli sample buffer (Bio-Rad catalog no. 161-0737).

Major content: $62.5 \mathrm{mM}$ Tris-HCl (pH: 6.8 ), 25\% glycerol, $2 \%$ SDS, and $0.01 \%$ Bromophenol blue.

Preparation 1 (Pre-1). Preparation 2 (Pre-2): ReadyPrep sequential extraction reagent 3 (Bio-Rad catalog no. 163-2104).

Major content: $5 \mathrm{M}$ urea, $2 \mathrm{M}$ thiourea, 2\% CHAPS, $2 \%$ SB 3-10, 40 mM Tris, and 0.2\% Bio-Lyte 3/10 ampholyte.

Five hundred microliters of elution buffer were added, and the column was spun for 5 seconds at 7,000 rpm in a microcentrifuge. The residual elution was collected and labeled as "serum protein."

Albumin fraction and amount of the serum protein samples were obtained by cellulose acetate strip electrophoresis. Briefly, Tris-barbital-sodium barbital buffer solution (Electra HR Buffer, Helena laboratories catalog no. 5805, TX, US) was added to the electrophoresis tank (SPIFE 3000, Helena Laboratories). Serum sample was applied to the plate (Titan III Cellulose Acetate, Helena laboratories catalog no. 3023) and electrophoresed at $180 \mathrm{~V}$ for 25 minutes. After the electrophoresis was done, the plates were removed from the electrophoresis chamber and stained (Ponceau S Stain, Helena Laboratories catalog no. 5526) for 5 minutes. A scanning densitometer (QuickScan 2000, Helena Laboratories) was employed for the estimation of the results of electrophoresis.
A serum sample without any extraction procedure (Pre3) was also examined as an internal control. The preparation group with the highest albumin fraction was chosen, and the radiolabeling procedures described below were performed.

\subsection{Preparation of the ${ }^{99 m} T c$-Labeled Autoalbumin. After} proper serum extraction of the albumin, radiolabeling was initiated. Sodium pertechnetate $\left({ }^{99 \mathrm{~m}} \mathrm{Tc}\right.$-pertechnetate) was eluted from an ${ }^{99} \mathrm{Mo} /{ }^{99 m} \mathrm{Tc}$ generator (Ultra-Techne Kow; Daiichi Radioisotope Laboratories, LTD., Tokyo, Japan), following manufacturer's instructions. Stannous solution (Amerscan Stannous Agent, Amersham plc, Buckinghamshire, UK) was prepared fresh by careful addition of $10 \mathrm{~mL} 0.9 \%$ sodium chloride to the kit's vial. The final concentration of the stannous fluoride in the stannous solution was $0.4 \mathrm{mg} / \mathrm{mL}$.

Labeling was carried out by mixing autoalbumin with the stannous solution and ${ }^{99 \mathrm{~m}} \mathrm{Tc}$-pertechnetate. Two different preparation protocols of ${ }^{99 \mathrm{~m}} \mathrm{Tc}$-autoalbumin were prepared, with (Protocol A) and without (Protocol B) stannous solution. First, $0.5 \mathrm{~mL}$ autoalbumin was measured and aliquoted into a sterile tube (BD Vacutainer; Becton, Dickinson and Company, NJ, US), then the stannous solution $(0.5 \mathrm{~mL})$ was added to the Protocol A test tubes using volumetric pipettes. After ${ }^{99 \mathrm{~m}} \mathrm{Tc}$-pertechnetate elution was completed; total activity was measured by a dose calibrator (CRC-15R; Capintec, Inc., NJ, US). 1.11 GBq of ${ }^{99 \mathrm{~m}}$ Tc-pertechnetate was collected and added with $0.9 \%$ sodium chloride to bring the final volume to $0.5 \mathrm{~mL}$, and then it was placed in a sterile tube. The autoalbumin and stannous mixtures were then transferred aseptically to the tubes containing ${ }^{99 \mathrm{~m}} \mathrm{Tc}$ pertechnetate using a syringe to carefully deliver the solution down the side wall of the tubes. Mixing was performed by gentle manual shaking to avoid bubble formation.

2.3. Radiochemical Purity and Stability. Radiochemical purity and stability were determined by instant thin-layer chromatography (ITLC). Initially, ITLC silica gel plate was cut into $0.8 \times 10 \mathrm{~cm}^{2}$ strips. Using a Hamilton syringe, one drop of the specimen was placed at $1.5 \mathrm{~cm}$ above the bottom of the strip. The strip was developed by acetone, and the radioactivity distribution over the strip was determined with a radio-TLC imaging scanner (AR-2000; Bioscan, Inc., Washington, DC, US). Radiochemical purity was calculated as the fraction of radioactivity that remained at the origin and was designated as \%RCP. After preparation, the stability of the mixture was acquired from sequential repeats $(n=6)$ of the $\% \mathrm{RCP}$ procedure at $10 \mathrm{~min}, 30 \mathrm{~min}, 1$ hour, 2 hours, 4 hours, 6 hours, and 24 hours, respectively.

2.4. Biodistribution. A gamma camera (DST-XLi, General Electric Medical Systems, Buc, France) was used to view rats (Wistar strain, male, weighing from 200 to $250 \mathrm{gm}$ ) that had been injected, via the tail vein, with $74 \mathrm{MBq}$ of ${ }^{99 \mathrm{~m}} \mathrm{Tc}-$ autoalbumin. The rats were anesthetized with an intraperitoneal injection of pentobarbital $(0.006 \mathrm{mg} / 100 \mathrm{gm})$. The gamma camera was fitted with a low-energy, high-resolution 
TABLE 1: Serum protein components separated by different elution buffers.

\begin{tabular}{lcccc}
\hline & Pre-1 & Pre- & Pre-3 & Reference [18] \\
\hline Total protein $(\mathrm{gm} / \mathrm{dL})$ & $2.00 \pm 0.16$ & $3.12 \pm 0.36$ & $7.26 \pm 0.19$ & $6.0-8.4$ \\
Electrophoresis (\%) & & & & \\
$\quad$ Albumin & $77.50 \pm 2.24$ & $4.36 \pm 1.15$ & $55.14 \pm 4.82$ & $52-68$ \\
Globulin $\alpha 1$ & $2.86 \pm 0.57$ & $2.08 \pm 0.62$ & $2.34 \pm 0.21$ & $4.2-7.2$ \\
Globulin $\alpha 2$ & $6.12 \pm 1.88$ & $87.02 \pm 2.52$ & $8.36 \pm 1.06$ & $6.8-12$ \\
Globulin $\beta$ & $7.04 \pm 1.41$ & $3.40 \pm 1.03$ & $13.36 \pm 1.52$ & $9.3-15$ \\
Globulin $\gamma$ & $6.48 \pm 3.23$ & $3.14 \pm 1.13$ & $20.80 \pm 3.53$ & $13-23$ \\
\hline
\end{tabular}

collimator, and the images were processed by a gammacamera working station (POWERstation SPX, IBM RS6000, Vision 5.2.0, General Electric Medical Systems). Each rat was studied by taking 750 frames of 0.4 seconds, followed by 1 frame of 60 seconds (total study time, $\sim 6$ minutes).

In addition to the imaging studies, an examination of biodistribution changes over time was performed. Rats were injected via tail vein. The animals $(n=5$, each group) were sacrificed by direct decapitation at $5,10,30,60,120$, and $240 \mathrm{~min}$ after administration of ${ }^{99 \mathrm{~m}} \mathrm{Tc}$-autoalbumin, followed by dissection. Organs and tissues were separated, and the radioactivity was determined using a gamma counter (COBRAII, Packard, Netherlands). Organ activity was expressed as a mean of percentage of injected dose per grams $(\% \mathrm{ID} / \mathrm{gm})$ of tissue.

2.5. Blood Clearance. Blood clearance studies were performed to understand the in vivo behavior of the injected ${ }^{99 \mathrm{~m}} \mathrm{Tc}$-autoalbumin. Rats $(n=5)$ without previous administration of any radiopharmaceutical were acquired. The radiolabeled preparations were diluted with $0.9 \%$ sodium chloride to a final concentration of $74 \mathrm{MBq} / \mathrm{mL}$, and $0.2 \mathrm{~mL}$ of this preparation was injected into the proximal portion of the tail vein. At fixed time intervals $(5,10,15,30,60$, and $120 \mathrm{~min}$ ), $0.2 \mathrm{~mL}$ blood samples were withdrawn from the distal end of the tail vein. All of the samples were counted in a gamma well counter and were compared with a value obtained for a standard radiopharmaceutical. The blood clearance rates were calculated by means of the logarithmic equation as follows:

$$
\% \text { Radioactivity, blood }=q \times[\ln (\text { Time })]+b .
$$

Time: minutes after injection, $q$ : slope, $b$ : intercept.

2.6. Pyrogenicity and Sterility Testing. Pyrogenicity was determined using a bacterial endotoxin test called the limulus amebocyte lysate (LAL) test. The sensitivity of the LAL reagent is 0.25 endotoxin units $(\mathrm{EU}) / \mathrm{mL}$. According to the requirement, as specified in USP32/NF27 [19], the limit with regard to endotoxin content of the ${ }^{99 \mathrm{~m}} \mathrm{Tc}$-autoalbumin preparation is $175 / \mathrm{V}$ USP EU/mL of the injection, where $\mathrm{V}$ is the maximum recommended dose in milliliters.

The sterility of the ${ }^{99 \mathrm{~m}} \mathrm{Tc}$-autoalbumin preparation was tested, as stated in USP32/NF27 [20]. One milliliter of our preparation was added to a test tube containing $15 \mathrm{~mL}$ of SCD and TGC medium. The tubes were observed for turbidity, and results were recorded every day for 14 days. A negative result was indicated by a clear culture medium (no turbidity noted) over the 14 days of observation.

2.7. Ethics. This study was approved by the Institutional Review Board of the Buddhist Dalin Tzu Chi General Hospital. All the animal experiments were performed in accordance with the Animal Protection Act of the Council of Agriculture, and were approved by the Institutional Animal Care and Use Committee of Dalin Tzu Chi General Hospital.

\section{Results}

3.1. Autoalbumin Fraction of the Elution. Five serum samples were prepared according to the protocol described above and were arranged into three groups: preparation 1 (Laemmli sample buffer; Pre-1), preparation 2 (ReadyPrep sequential extraction reagent 3; Pre-2), and preparation 3 (serum without any extraction; Pre-3). The Pre-3 served as an internal control for the electrophoresis analysis, and our data showed evenly distributed protein elements (Table 1) upon comparison to the reference range [18], with the exception of a slightly lower fraction than the globulin $\alpha 1$. Both Pre- 1 and Pre-2 exhibited diminished total protein concentrations (2.00 gm/dL and $3.12 \mathrm{gm} / \mathrm{dL}$, resp.) as compared to Pre-3 (7.62 gm/dL), which was caused by inevitable loss from the extraction procedure. Pre-1 yielded a higher extraction ratio of the albumin fraction from the serum protein $(77.5 \%)$ as compared with Pre-2 (4.36\%) and Pre-3 (55.14\%) while the Pre-2 showed a high fraction in the globulin $\alpha 2(87.02 \%)$ then Pre-1 (6.12\%) and Pre-3 (8.36\%) (Table 1). This result indicated that Pre-1, the extraction serum with Laemmli sample buffer, effectively isolated a higher proportion of autoalbumin. All of the radiolabeling studies were performed with high-purity autoalbumin from Pre-1.

3.2. Radiochemical Purity and Stability. Protocol A contained $0.5 \mathrm{~mL}$ autoalbumin, $0.5 \mathrm{~mL}$ stannous solution, and $1.11 \mathrm{GBq}$ of ${ }^{99 \mathrm{~m}} \mathrm{Tc}$-pertechnetate. Protocol B contained $0.5 \mathrm{~mL}$ autoalbumin with $1.11 \mathrm{GBq}$ of ${ }^{99 \mathrm{~m}} \mathrm{Tc}$-pertechnetate. Figure 1 demonstrates that the radiochemical purity obtained with Protocol A was $95.82 \%$ at $10 \mathrm{~min}$ and maintained the $94.42 \%$ out to 6 hours after preparation. Twenty-four hours after radiolabeling, the average RCP still reached $72.77 \%(n=6)$. Protocol B, however, resulted in substantially poorer labeling efficiency and was deemed unsuitable for clinical application. 

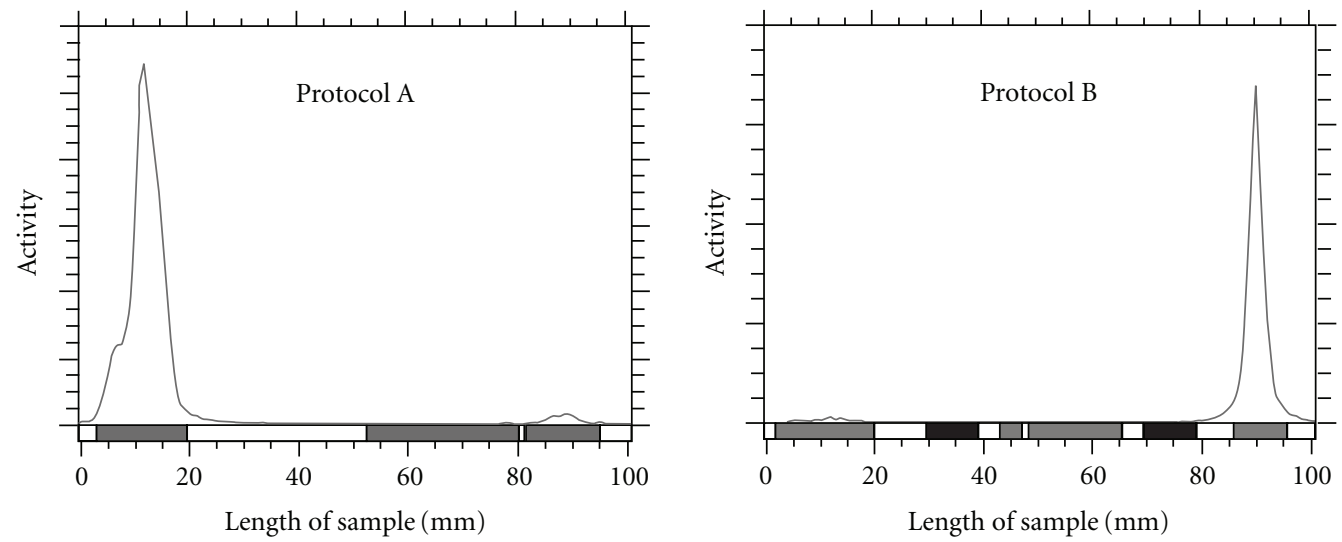

(a)

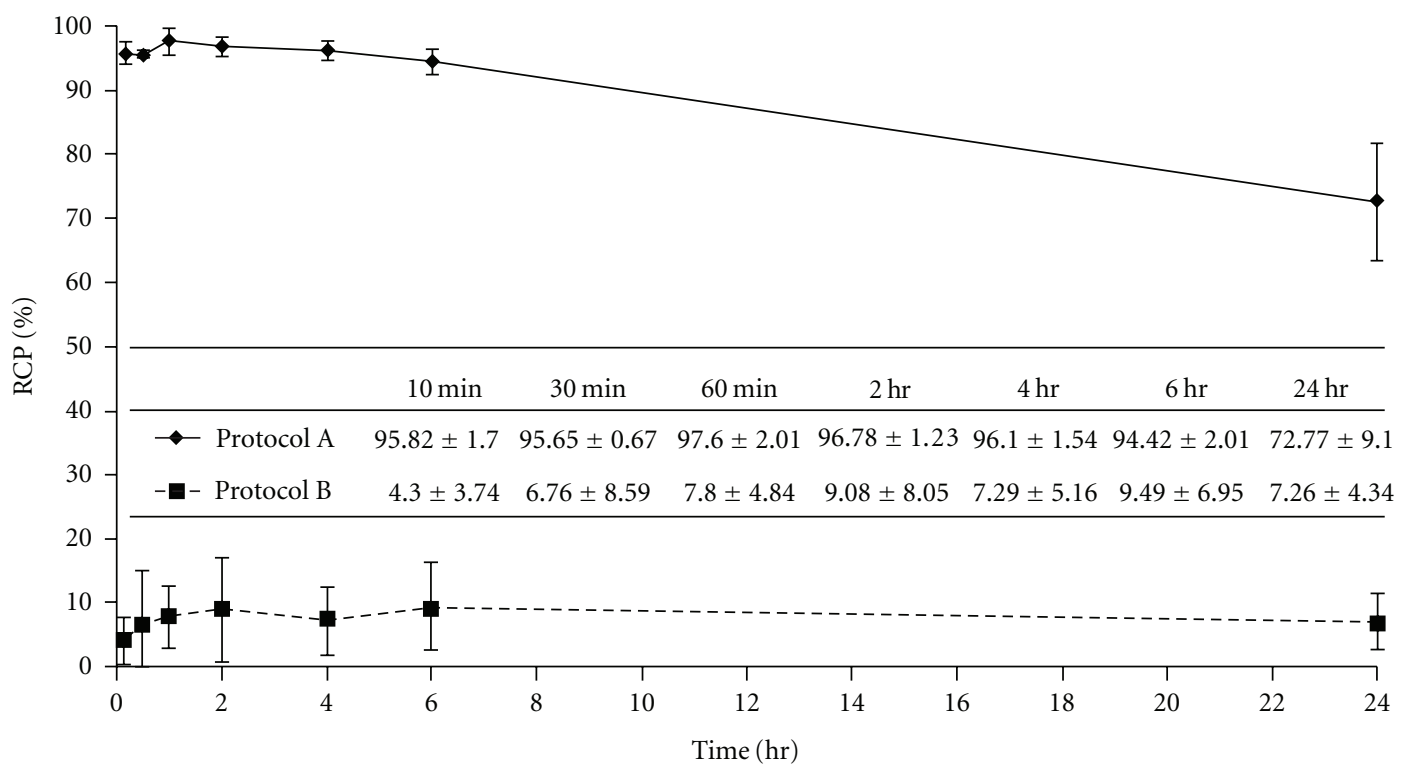

(b)

Figure 1: (a) Examples of the radiochemical purity studies from Protocol A and Protocol B. Both showed the RCP result of 30 min after radiolabeling. Radiochromatograms were acquired with a radio-TLC scanner. (b) Radiochemical purity and stability tests of the preparation protocols, determined by ITLC and presented as \% RCP with mean $\pm \mathrm{SD}(n=6)$.

TABLE 2: In vivo biodistribution of the ${ }^{99 \mathrm{~m}} \mathrm{Tc}$-autoalbumin, expressed as percentage of administered dose per gram of organ (\%ID/gm) (mean values \pm s.e.m.).

\begin{tabular}{|c|c|c|c|c|c|c|c|c|}
\hline Time (min) & Brain & Lung & Liver & Stomach & Spleen & Kidney & Muscle & Bone \\
\hline 5 & $0.14 \pm 0.09$ & $0.93 \pm 0.60$ & $1.46 \pm 0.63$ & $0.43 \pm 0.23$ & $0.48 \pm 0.20$ & $2.91 \pm 0.72$ & $0.19 \pm 0.10$ & $0.08 \pm 0.02$ \\
\hline 10 & $0.23 \pm 0.13$ & $1.03 \pm 0.64$ & $1.27 \pm 0.25$ & $0.57 \pm 0.12$ & $0.73 \pm 0.21$ & $2.44 \pm 1.10$ & $0.64 \pm 0.13$ & $0.23 \pm 0.10$ \\
\hline 30 & $0.02 \pm 0.01$ & $0.09 \pm 0.02$ & $0.94 \pm 0.52$ & $0.22 \pm 0.08$ & $0.30 \pm 0.12$ & $3.37 \pm 0.95$ & $0.06 \pm 0.01$ & $0.05 \pm 0.01$ \\
\hline 60 & $0.01 \pm 0.01$ & $0.04 \pm 0.01$ & $0.76 \pm 0.11$ & $0.07 \pm 0.01$ & $0.13 \pm 0.07$ & $3.29 \pm 0.86$ & $0.12 \pm 0.03$ & $0.07 \pm 0.01$ \\
\hline 120 & $0.02 \pm 0.02$ & $0.09 \pm 0.03$ & $0.70 \pm 0.24$ & $0.15 \pm 0.08$ & $0.30 \pm 0.09$ & $4.48 \pm 0.39$ & $0.03 \pm 0.01$ & $0.07 \pm 0.02$ \\
\hline 240 & $0.01 \pm 0.01$ & $0.05 \pm 0.02$ & $0.52 \pm 0.13$ & $0.11 \pm 0.03$ & $0.52 \pm 0.17$ & $4.12 \pm 0.69$ & $0.04 \pm 0.03$ & $0.05 \pm 0.02$ \\
\hline
\end{tabular}

3.3. Biodistribution. When the ${ }^{99 \mathrm{~m}} \mathrm{Tc}$-autoalbumin, prepared as Protocol A, was injected intravenously into a rat, the main vessels, heart, lung, liver, and kidneys were immediately visualized (Figures 2(a) and 2(b)). Results from the animal biodistribution studies were expressed as percentages of injected doses per gram and presented in Table 2. Five minutes after initiation of the study, a percentage of the injected dose of radiolabeled autoalbumin was detected in the lung $(0.93 \%)$, liver $(1.46 \%)$, and kidneys $(2.91 \%)$. The distributions to the lung (1.04\%) and liver $(1.27 \%)$ were highest at 10 minutes after intravenous injection of the radiotracer. These levels were reduced in lung and liver, but 


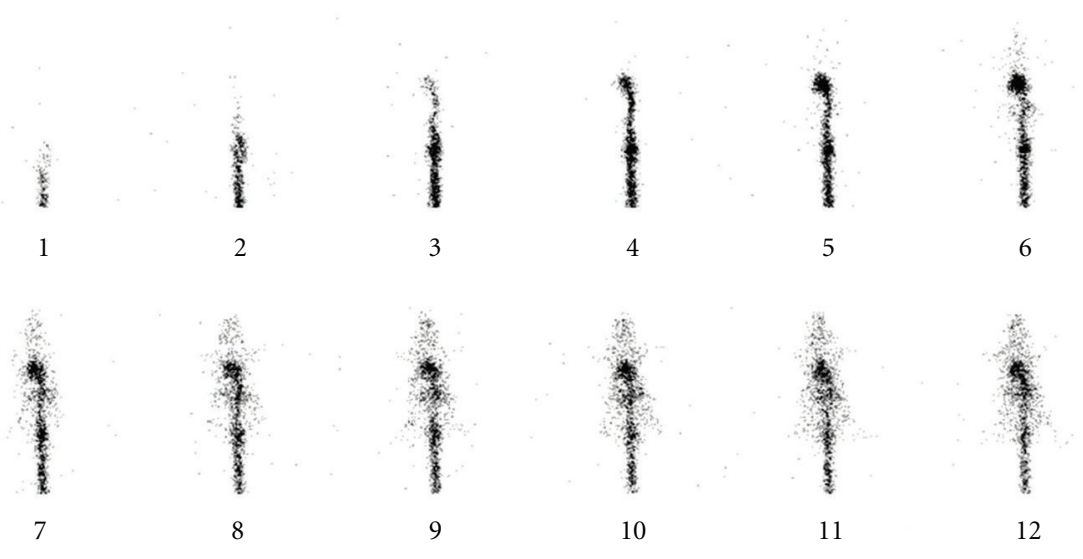

(a)

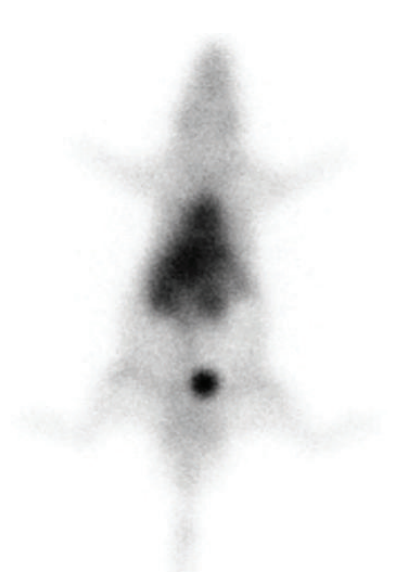

(b)

FIGURE 2: Image studies for the biodistribution of the ${ }^{99 \mathrm{~m}} \mathrm{Tc}$-autoalbumin. (a) Dynamic images, rats were studied by taking 750 frames of 0.4 seconds interval; (b) static image taken at $5 \mathrm{~min}$ after injection with an acquisition time of 60 seconds.

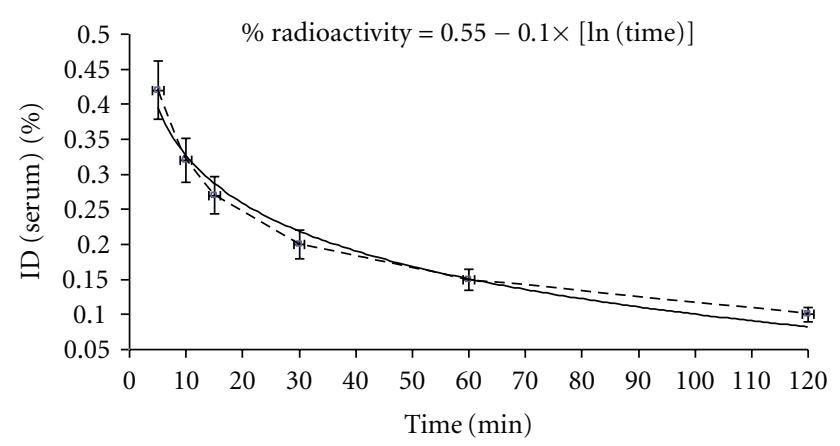

FIGURE 3: Blood clearance of the ${ }^{99 \mathrm{~m}} \mathrm{Tc}$-autoalbumin in study animals $(n=5)$.

in the kidneys gradually increased to $4.48 \%$ over the first 2 hours. The radiodistribution of the stomach and other internal organs, such as spleen, showed no apparent difference (stomach: $0.57 \%$ to $0.11 \%$; spleen: $0.73 \%$ to $0.13 \%$ ).

3.4. Blood Clearance. The serum elimination of this radiolabeled autoalbumin has been shown in Figure 3. A decline in percentage of injected dose in blood was observed over time. The formula used to calculate the blood clearance is "\%Radioactivity $=0.55-0.1 \times[\ln ($ Time $)] "\left(r^{2}>0.95\right)$, and the biological half-life calculated was 32.29 minutes.

3.5. Pyrogenicity and Sterility Testing. After 6 hours, remaining ${ }^{99 \mathrm{~m}} \mathrm{Tc}$-autoalbumin was used to perform pyrogen and sterility tests $(n=5)$. In the pyrogenicity tests, those samples diluted 50 -fold and 83 -fold (the maximum valid dilution value) yielded negative results. In addition, after being incubated for 14 days, our culture medium remained clear in appearance. All samples were found to be sterile and pyrogen-free.

\section{Discussion}

The term autologous transfusion describes any procedure by which donated blood (or serum component) is transfused (or reinfused) into the donor/patient [21]. The primary purpose of autologous transfusion is to prevent exposure to blood-borne infectious agents. This has become increasingly critical as awareness of the risks of allergenic blood products has grown in the decades following the AIDS epidemic that plagued the late 1980s and early 1990s.

The first step of this study was designed to establish an autoalbumin extraction procedure from single-source, autologous serum. Specifically, the serum sample was placed in a resin bead column to capture the protein component. Upon release from the column, total protein and separated component fractions were examined by electrophoresis. An internal control sample provided the reference range of serum protein distribution, with the exception of a relatively lower fraction of the globulin $\alpha 1$. Elution by Laemmli sample buffer yielded a high albumin elution fraction (77.5\%) while elution by ReadyPrep sequential extraction reagent 3 yielded a higher fraction in the globulin $\alpha 2$. Serum samples subject to resin bead column isolation and elution by Laemmli sample buffer were determined to be suitable for use with the subsequent radiolabeling procedure.

Radiolabeling was performed by carefully mixing ${ }^{99 \mathrm{~m}} \mathrm{Tc}$ pertechnetate and autoalbumin. Stannous is essential for good radiolabeling efficiency. Our data showed that the radiochemical purity and stability were sufficient, because 6 hours after radiolabeling, the \%RCP still reached more than $94 \%$. When the radiolabeled autoalbumin was injected intravenously into a rat, the lung, liver, and kidneys were promptly visualized. The distribution to the lung and liver was highest within the initial 10 minutes and then vanished gradually. In the kidney, the accumulation of radioactivity became progressively prominent, suggesting the major excretory pathway of radiolabeled autoalbumin may be the genitourinary tract. The radioactivity to the gastric mucosa showed no 
significant difference as other internal organs, for example, spleen, could be an in vivo demonstration of stable labeling of Technetium-99m and autoalbumin in study animals.

A previous literature report of the biodistribution in rat with ${ }^{99 \mathrm{~m}} \mathrm{Tc}-\mathrm{HSA}$ demonstrates a rapid accumulation of tracer to the liver immediately after injection while the timeactivity cure showed slightly decreased radio-uptake with time [22]. Excretion of tracer through the kidneys could be also identified right after injection of tracer. Another study described the biodistribution of the recombinant human serum albumin (rHSA) also showed major initial distribution to the liver [23]. However, progressive decrement of liver uptake and increment of renal activity were also observed. In this study, time series sacrifices and dissection of organs/tissues were performed. Our results were consistent with these reports for the biodistribution study.

Autologous blood and blood components are widely believed to be the safest blood products available for clinical application; however, certain complications are still possible and should be kept under a strict watch. Contamination by bacteria or other pyrogens during the preparation steps remain a risk. Our autoalbumin preparation procedure was determined to be free from pyrogenicity and passed sterility tests; however, operator errors or negligence may still occur. Prevention of contamination is of ultimate importance in clinical practice of nuclear medicine. Another operator error that may occur is mislabeling of serum tubes. This mistake yields the identical risks associated with nonautologous transfusion and can lead to serum product administration to an incompatible patient.

Autologous preparations of blood component techniques are generally intended to replace as much of the allogenic origin as possible by reinfusion of autologous blood products. Our study demonstrates that the separation of autoalbumin takes no longer than 2 hours and radiolabeling may be carried out immediately after separation. This implies that the collection and reprocessing of radiolabeled autoalbumin and the subsequent nuclear medical procedure could be performed as a daily practice. Even though ${ }^{99 \mathrm{~m}} \mathrm{Tc}$-albumin labeling has demonstrated some clinical advantages, it is still a significantly hazardous procedure. Therefore, it may not be worth the risk if a kit formulation is available to be used.

The most recent development of nonserum sources of human albumin has been the rHSA [23-26]. Both rHSA and pHSA have been shown to be identical in structure and physiochemical and immunochemical properties $[27,28]$. In the future, rHSA promises to be an ideal source for a "clean" HSA.

\section{Conclusions}

Radiolabeled human serum albumin is an important nuclear medicine radiopharmaceutical. However, the safety of pooled serum products remains a concern. Our study demonstrates that autoalbumin can be efficiently extracted within a nuclear medicine laboratory and immediately radiolabeled. This radiolabeled autoalbumin exhibited sufficient radiochemical purity and stability. The most important advantage to this technique is that the risk of infection could be significantly eliminated by this preparation of autoalbumin.

\section{References}

[1] C. Vanhove, N. Walgraeve, F. De Geeter, and P. R. Franken, "Gated myocardial perfusion tomography versus gated blood pool tomography for the calculation of left ventricular volumes and ejection fraction," European Journal of Nuclear Medicine and Molecular Imaging, vol. 29, no. 6, pp. 735-741, 2002.

[2] S. Ferraro, P. Perrone-Filardi, A. Desiderio et al., "Left ventricular systolic and diastolic function in severe obesity: A radionuclide study," Cardiology, vol. 87, no. 4, pp. 347-353, 1996.

[3] N. T. Chiu, B. F. Lee, S. J. Hwang, J. M. Chang, G. C. Liu, and H. S. Yu, "Protein-losing enteropathy: diagnosis with (99m)Tc-labeled human serum albumin scintigraphy," Radiology, vol. 219, no. 1, pp. 86-90, 2001.

[4] I. Bedrosian, A. M. Scheff, R. Mick et al., " $99 m$ Tc-human serum albumin: an effective radiotracer for identifying sentinel lymph nodes in melanoma," Journal of Nuclear Medicine, vol. 40, no. 7, pp. 1143-1148, 1999.

[5] Y. C. Chen, S. J. Hwang, J. S. Chiu, M. H. Chuang, M. I. Chung, and Y. F. Wang, "Chronic edema from protein-losing enteropathy: scintigraphic diagnosis," Kidney International, vol. 75 , no. 10, p. 1124, 2009.

[6] R. Kim, A. Osaki, J. Kojima, and T. Toge, "Significance of lymphoscintigraphic mapping with Tc-99m human serum albumin and tin colloid in sentinel lymph node biopsy in breast cancer," International journal of oncology, vol. 19, no. 5, pp. 991-996, 2001.

[7] M. Momose, S. Kawakami, T. Koizumi et al., "Lymphoscintigraphy using technetium-99m HSA-DTPA with SPECT/CT in chylothorax after childbirth," Radiation Medicine, vol. 26, no. 8, pp. 508-511, 2008.

[8] B. R. Don and G. Kaysen, "Serum albumin: relationship to inflammation and nutrition," Seminars in Dialysis, vol. 17, no. 6, pp. 432-437, 2004.

[9] L. T. Goodnough, "Risks of blood transfusion," Critical Care Medicine, vol. 31, no. 12, pp. S678-S686, 2003.

[10] C. Madjdpour, V. Heindl, and D. R. Spahn, "Risks, benefits, alternatives and indications of allogenic blood transfusions," Minerva Anestesiologica, vol. 72, no. 5, pp. 283-298, 2006.

[11] W. R. Mayr, “The reality of self-sufficiency," Transfusion Clinique et Biologique, vol. 12, no. 5, pp. 362-364, 2005.

[12] The President's Emergency Plan for AIDS Relief, "Report on blood safety and HIV/AIDS," June 2006, http://www.pepfar .gov/progress/76858.htm.

[13] Centers for Disease Control and Prevention (CDC), "Progress toward strengthening blood transfusion services-14 countries, 2003-2007," Morbidity and Mortality Weekly Report, vol. 57, no. 47, pp. 1273-1277, 2008.

[14] Y. F. Wang, M. H. Chuang, J. S. Chiu, T. M. Cham, and M. I. Chung, "On-site preparation of technetium-99m labeled human serum albumin for clinical application," Tohoku Journal of Experimental Medicine, vol. 211, no. 4, pp. 379-385, 2007.

[15] L. T. Goodnough, M. E. Brecher, M. H. Kanter, and J. P. Aubuchon, "Transfusion medicine: II. Blood conservation," New England Journal of Medicine, vol. 340, no. 7, pp. 525-533, 1999. 
[16] R. Karger and V. Kretschmer, "Modern concepts of autologous haemotherapy," Transfusion and Apheresis Science, vol. 32, no. 2, pp. 185-196, 2005.

[17] E. Gianazza and P. Arnaud, "Chromatography of plasma proteins on immobilized Cibacron Blue F3-GA. Mechanism of the molecular interaction," Biochemical Journal, vol. 203, no. 3, pp. 637-641, 1982.

[18] "Case records of the Massachusetts General Hospital. Weekly clinicopathological exercises. Normal reference values," The New England Journal of Medicine, vol. 314, no. 1, pp. 39-19, 1986.

[19] U.S. Pharmacopeia, "Bacterial endotoxin test," in USP32/ NF27: United States Pharmacopeia and National Formulary, pp. 93-96, The United States Pharmacopeial Convention Inc., 2009.

[20] U.S. Pharmacopeia, "Sterility test," in USP32/NF27: United States Pharmacopeia and National Formulary, pp. 80-86, The United States Pharmacopeial Convention Inc., 2009.

[21] P. T. Toy, R. G. Strauss, and L. C. Stehling, "Predeposited autologous blood for elective surgery. A national multicenter study," New England Journal of Medicine, vol. 316, no. 9, pp. 517-520, 1987.

[22] K. Kristensen, "Biodistribution in rats of 99mTc-labelled human serum albumin," Nuclear Medicine Communications, vol. 7, no. 8, pp. 617-624, 1986.

[23] A. C. Perkins and M. Frier, "Experimental biodistribution studies of $99 \mathrm{mTc}$-recombinant human serum albumin (rHSA): a new generation of radiopharmaceutical," European Journal of Nuclear Medicine, vol. 21, no. 11, pp. 1231-1233, 1994.

[24] S. Matsushita, Y. Isima, V. T. Chuang et al., "Functional analysis of recombinant human serum albumin domains for pharmaceutical applications," Pharmaceutical Research, vol. 21, no. 10, pp. 1924-1932, 2004.

[25] K. Kobayashi, "Summary of recombinant human serum albumin development," Biologicals, vol. 34, no. 1, pp. 55-59, 2006.

[26] K. Langer, M. G. Anhorn, I. Steinhauser et al., "Human serum albumin (HSA) nanoparticles: reproducibility of preparation process and kinetics of enzymatic degradation," International Journal of Pharmaceutics, vol. 347, no. 1-2, pp. 109-117, 2008.

[27] A. Kasahara, K. Kita, E. Tomita, J. Toyota, Y. Imai, and H. Kumada, "Repeated administration of recombinant human serum albumin caused no serious allergic reactions in patients with liver cirrhosis: a multicenter clinical study," Journal of Gastroenterology, vol. 43, no. 6, pp. 464-472, 2008.

[28] W. Ohtani, Y. Nawa, K. Takeshima, H. Kamuro, K. Kobayashi, and T. Ohmura, "Physicochemical and immunochemical properties of recombinant human serum albumin from Pichia pastoris," Analytical Biochemistry, vol. 256, no. 1, pp. 56-62, 1998. 


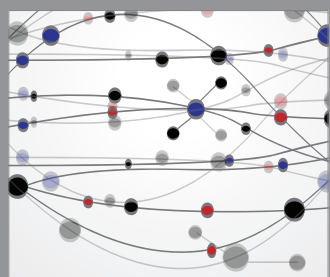

The Scientific World Journal
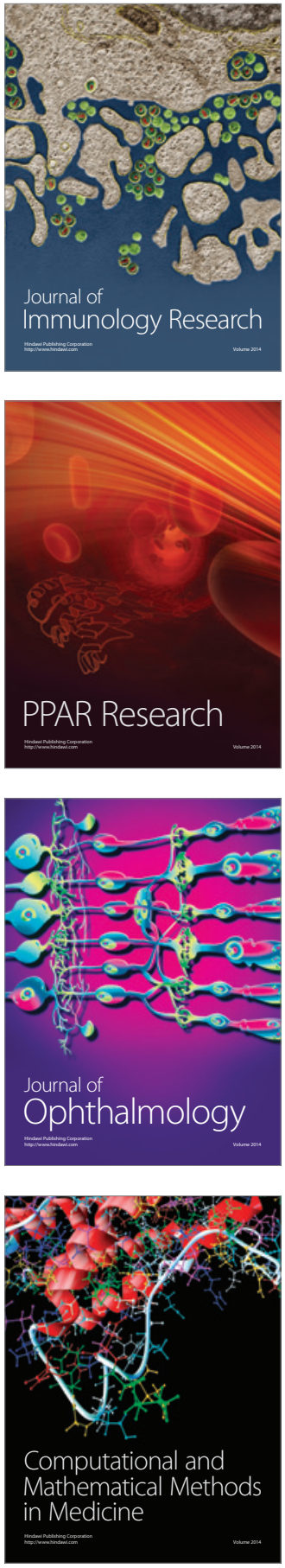

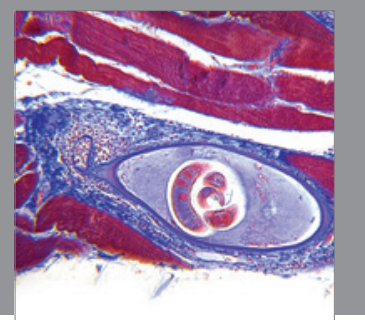

Gastroenterology

Research and Practice
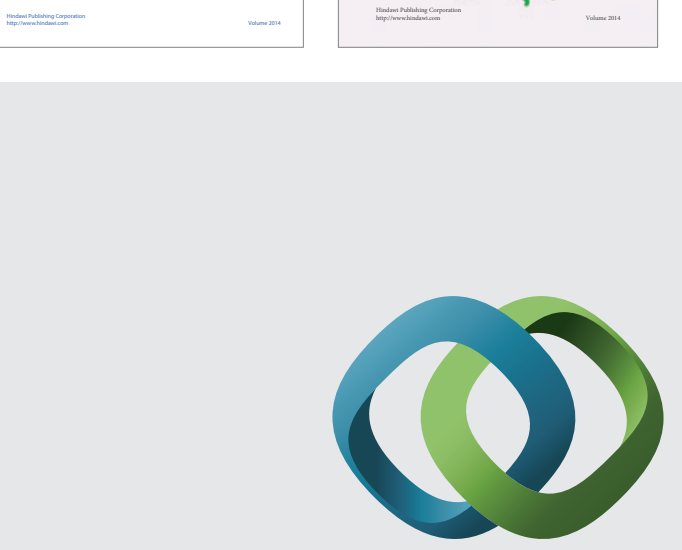

\section{Hindawi}

Submit your manuscripts at

http://www.hindawi.com
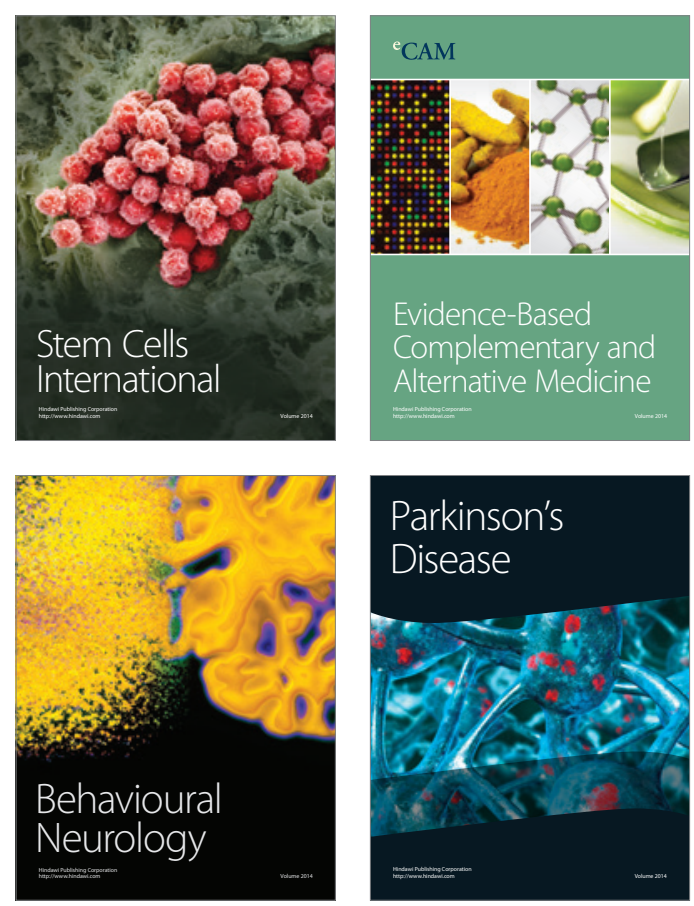

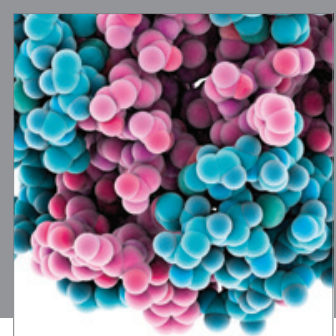

Journal of
Diabetes Research

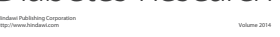

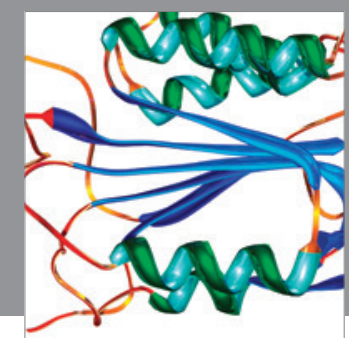

Disease Markers
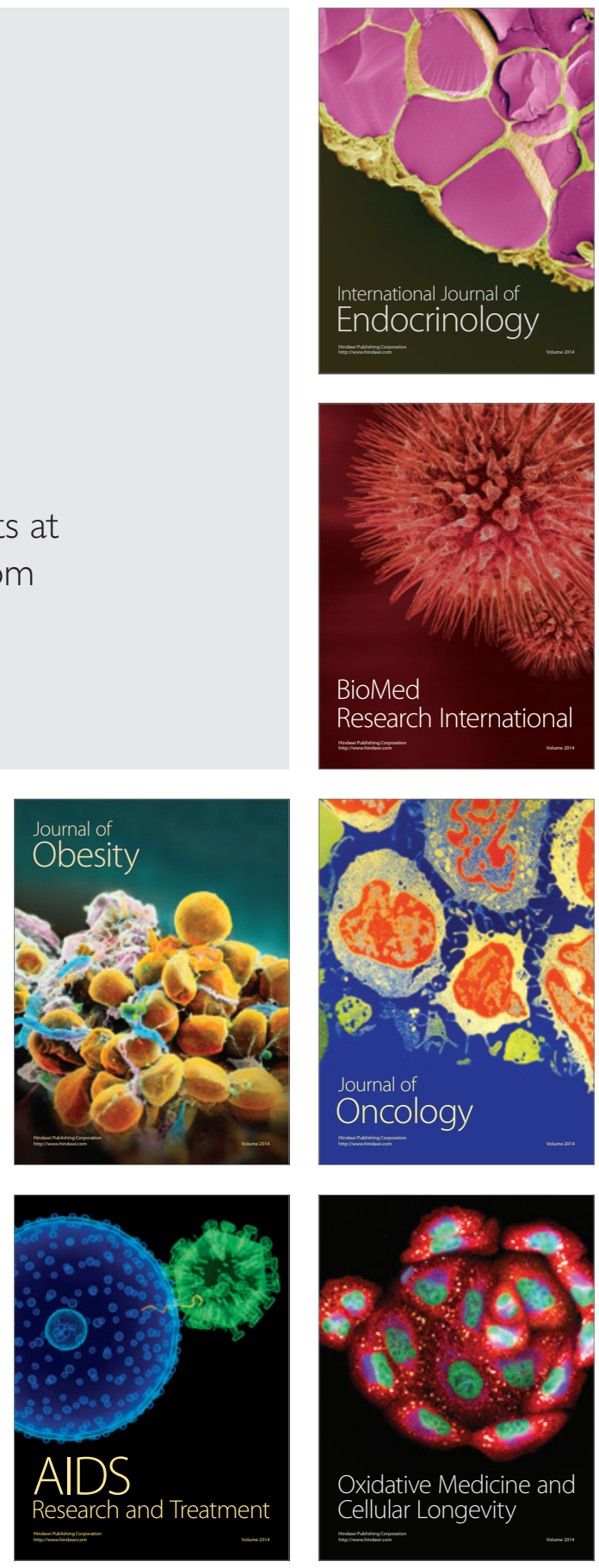